\title{
Therapeutic Options for the Treatment of Carbapenem- resistant Enterobacteriaceae Infections: Hope in the Times of Hype and Despair
}

\author{
Abdul S Ansario \\ Indian Journal of Critical Care Medicine (2021): 10.5005/jp-journals-10071-23918
}

"May your choices reflect your hopes, not your fears"-Nelson Mandela

\section{BACKGROUND}

Antimicrobial resistance is the most imminent threat to public health, and in the absence of an effective antimicrobial stewardship program, it threatens our healthcare delivery system to an unprecedented level of disruption. ${ }^{1,2}$

Acquisition of hospital-acquired infections is affected by a multitude of factors like underlying comorbidities, immune status, therapeutic interventions, prior antimicrobial exposure, and hospital infection control practices. ${ }^{3,4}$ Among all the multidrugresistant organisms, carbapenem-resistant Enterobacteriaceae (CRE) are of the highest concern due to their rising incidence and associated mortality. ${ }^{5}$

\section{Current Evidence}

Barring a few consensus statements, there is a serious paucity of highlevel evidence on managing CRE infections and an urgent need to find effective drugs in the post-carbapenem era. ${ }^{6}$ Most CRE organisms have multiple resistance mechanisms (Ambler class $A, B$, and D), and a rapid screening for genetic mechanisms is possible with either conventional multiplex PCR or Xpert Carba-R assay. ${ }^{7,8}$ Coproduction of NDM-1 with OXA-48 has been increasingly encountered in our intensive care units (ICUs) along with frequent possession of 16S rRNA methylase gene in NDM-producing Enterobacteriaceae. ${ }^{9-11}$ In light of this, it becomes imperative for the clinician to use combination therapies in seriously ill patients with CRE infections as an initial inappropriate antibiotic regime has been associated with increased mortality or prolonged ICU stay. ${ }^{12}$ Polymyxins ( $B$ and $E$ ) have exhibited their superiority for the treatment of CRE infections and remain the cornerstone molecule for both empiric monotherapy and combination regimes. ${ }^{13,14}$ A preference for combination therapy in patients with high mortality risk score [INCREMENT-CPE score (ICS)] or worsening SOFA scores $(>2)$ would be the right strategy in NDMprevalent Indian setting. ${ }^{15,16}$ Also, tigecycline and fosfomycin have shown considerable potential to counter CRE menace when used in combination regimes based on site of infection, PK/PD characteristics, and molecular resistance phenotypes.

Novel anti-infectives like CZA (ceftazidime + avibactam) or MER-VAB (meropenem + vaborbactam) do not provide cover against NDM which seriously limits their utility in Indian setting. ${ }^{17-19}$ Although CZA salvage therapy for severe KPC (Klebsiella pneumoniae carbapenemase)
Department of Critical Care, Balabhai Nanavati Hospital, Mumbai, Maharashtra, India

Corresponding Author: Abdul S Ansari, Department of Critical Care, Balabhai Nanavati Hospital, Mumbai, Maharashtra, India, Phone: +91 9820812062, e-mail: drabdulansari@yahoo.co.in

How to cite this article: Ansari AS. Therapeutic Options for the Treatment of Carbapenem-resistant Enterobacteriaceae Infections: Hope in the Times of Hype and Despair. Indian J Crit Care Med 2021;25(7):752-753.

Source of support: Nil

Conflict of interest: None

infections including bacteremia has been well established, its utility would be of limited import for our CRE infections. ${ }^{20}$

\section{The Study in the Current Issue}

In this issue of the journal, a retrospective observational study taken up between February 2019 and January 2020 at two tertiary care centers has evaluated "Clinical outcomes of patients on CZA and combination therapy in CRE infections" with focus on underlying resistance mechanism and clinical cure rates ${ }^{21}$. The authors have explored the feasibility of CZA as monotherapy or in combination with AZT (aztreonam) and other agents based on the predominant phenotype of the CRE isolates. They have also documented a "Zone of Hope" by stacking CZA and AZT discs and/or MIC strips in close approximation to witness synergy and clearing zones of more than $18 \mathrm{~mm}$. Out of a total $121 \mathrm{CRE}$ isolates examined by Xpert Carba-R for rapid detection of gene sequences, only 57 patients were included for treatment comparison. Majority of the tested isolates were Klebsiella pneumoniae (83\%) while remaining strains were Escherichia coli (17\%) with OXA-48 (33\%) and NDM (28\%) being the dominant mechanisms of resistance. Nearly half of all the Klebsiella isolates were NDM + OXA-48 coproducers. There was a high degree of acute illness in the studied populations with majority of patients admitted in ICU (72\%). Majority of them were afflicted with intraabdominal (31.5\%) and nosocomial pneumonia (26.3\%) as the leading source of infection. CZA was used for OXA-48 producers either alone $(23 \%)$ or in combination $(77 \%)$ with other agents like polymyxin, tigecycline, or fosfomycin. For all NDM or NDM + OXA-48 coproducers, CZA + AZT was used in 30\% of patients as synergistic combination while another agent like polymyxin

(0) Jaypee Brothers Medical Publishers. 2021 Open Access This article is distributed under the terms of the Creative Commons Attribution 4.0 International License (https://creativecommons.org/licenses/by-nc/4.0/), which permits unrestricted use, distribution, and non-commercial reproduction in any medium, provided you give appropriate credit to the original author(s) and the source, provide a link to the Creative Commons license, and indicate if changes were made. The Creative Commons Public Domain Dedication waiver (http://creativecommons.org/publicdomain/zero/1.0/) applies to the data made available in this article, unless otherwise stated. 
or fosfomycin was added to the remaining $70 \%$ of subjects. An overall clinical cure rate without relapse or death (within 30 days) was reported in $78 \%$ of patients with a crude mortality of around $21 \%$ in the studied population.

A similar retrospective study published recently from our country studied the impact of CZA in clinical outcomes of CRE infections in a total of 103 patients with a matching clinical cure rate of $73 \%$ with all-cause mortality of $27 \%$. They reported an impressive 79\% sensitivity to CZA on E-tests (Epsilometer test), demonstrating its feasibility as a viable therapeutic option though no association was identified between E-test sensitivity and overall mortality (20). The study in consideration has tried to offer real-world data and highlights the burden of serious infection especially Klebsiella species (NDM + OXA-48 coproducers) as the "Thanos" of CRE era. In conjunction with other established antimicrobial agents, CZA + AZT can rise to the occasion as a possible therapeutic option in an algorithmic-MIC-based approach against the rising menace of CRE. To name a few shortcomings, there appears an overgeneralization of outcome, lack of homogeneity, survivorship bias (the treatment arm comprised less than half of CRE isolates studied), and a quick estimate of the potential "Zone of Hope."

\section{Author's View}

There is no hiding from the growing burden of CRE in Indian tertiary healthcare setting, the need for identification of resistance mechanisms, and the growing utility of combination therapies (cornerstone antibiotic with companion/adjuvant drugs) in treating this subset of critical patients. Further research with high-quality prospective/retrospective studies, case series, and meta-analyses to address this menace is a pressing priority.

\section{ORCID}

Abdul S Ansari ৫ib https://orcid.org/0000-0003-1015-1673

\section{References}

1. www.who.int.

2. www.cdc.gov.

3. Ghanshani R, Gupta R, Gupta BS, Kalra S, Khedar RS, Sood S. Epidemiological study of prevalence, determinants, and outcomes of infections in medical ICU at a tertiary care hospital in India. Lung India 2015;32(5):441. DOI: 10.4103/0970-2113.164155.

4. Ewans $T M$, Ortiz CR, LaForce FM. Prevention and control of nosocomial infection in the intensive care unit. In: Irwin RS, Cerra FB, Rippe JM, editors. Intensive care medicine. 4th ed. New York: Lippincot-Ravan, 1999, pp. 1074-1080.

5. Ramasubramanian V, Porwal R, Rajesh N. Carbapenem resistant Gram-negative bacteremia in an Indian intensive care unit: a review of the clinical profile and treatment outcome of 50 patients. Indian J Crit Care Med 18(11):750-753. DOI: 10.4103/0972-5229.144021.

6. Soman R, Veeraraghavan B, Hegde A, Jiandani P, Mehta $Y$, Nagavekar $V$, et al. Indian consensus on the management of CRE infection in critically ill patients (ICONIC) - India. Expert Rev Anti Infect Ther 2019;17(8):647-660. DOI: 10.1080/14787210.2019.1647103.

7. Yamamoto M, Pop-Vicas AE. Treatment for infections with carbapenem-resistant Enterobacteriaceae: what options do we still have? Crit Care 2014;18(3):229. DOI: 10.1186/cc13949.

8. Anandan S, Damodaran S, Gopi R, Bakthavatchalam YD, Veeraraghavan B. Rapid screening for carbapenem resistant organisms: current results and future approaches. JCDR 2015;9(9):DM01. DOI: 10.7860/JCDR/2015/14246.6530.

9. Veeraraghavan B, Shankar C, Karunasree S, Kumari S, Ravi R, Ralph R. Carbapenem resistant Klebsiella pneumoniae isolated from bloodstream infection: Indian experience. Pathog Glob Health 2017;111(5):240-246. DOI: 10.1080/20477724.2017.1340128.

10. Kazi M, Khot R, Shetty $A$, Rodrigues $C$. Rapid detection of the commonly encountered carbapenemases (New Delhi metallo-ßlactamase, OXA-48/181) directly from various clinical samples using multiplex real-time polymerase chain reaction assay. Indian J Med Microbiol 2018;36:369-375. DOI: 10.4103/ijmm.IJMM_18_324.

11. Wangkheimayum J, Paul D, Dhar D, Nepram R, Chetri S, Bhowmik D, et al. Occurrence of acquired $16 \mathrm{~S}$ rRNA methyltransferase-mediated aminoglycoside resistance in clinical isolates of Enterobacteriaceae within a tertiary referral hospital of Northeast India. Antimicrob Agents Chemother 2017;61(6):e01037. DOI: 10.1128/AAC.01037-16

12. Morrill HJ, Pogue JM, Kaye KS, LaPlante KL. Treatment options for carbapenem-resistant Enterobacteriaceae infections. Open Forum Infect Dis 2015;2:ofv050. DOI: 10.1093/ofid/ofv050.

13. Ni W, Cai X, Wei C, Di X, Cui J, Wang R, et al. Efficacy of polymyxins in the treatment of carbapenem-resistant Enterobacteriaceae infections: a systematic review and meta-analysis. Braz J Infect Dis 2015;19:170-180. DOI: 10.1016/j.bjid.2014.12.004.

14. Tsuji BT, Pogue JM, Zavascki AP, Paul M, Daikos GL, Forrest A, et al. International consensus guidelines for the optimal use of the polymyxins: endorsed by the American College of Clinical Pharmacy (ACCP), European Society of Clinical Microbiology and Infectious Diseases (ESCMID), Infectious Diseases Society of America (IDSA), International Society for Anti-infective Pharmacology (ISAP), Society of Critical Care Medicine (SCCM), and Society of Infectious Diseases Pharmacists (SIDP). Pharmacotherapy 2019;39(1):10-39. DOI: 10.1002/phar.2209.

15. Gutiérrez-Gutiérrez B, Salamanca E, de Cueto $M$, Hsueh P-R, Viale $P$, Paño-Pardo JR, et al. Effect of appropriate combination therapy on mortality of patients with bloodstream infections due to carbapenemase-producing Enterobacteriaceae (INCREMENT): a retrospective cohort study. Lancet Infect Dis 2017;17:726-734. DOI: 10.1016/S1473-3099(17)30228-1.

16. Shaw E, Rombauts A, Tubau F. Clinical outcomes after combination treatment with ceftazidime/avibactam and aztreonam for NDM-1/ OXA-48/CTX-M-15-producing Klebsiella pneumoniae infection. J Antimicrob Chemother 2018;73(4):1104-1106. DOI: 10.1093/jac/ dkx496.

17. Marshall S, Hujer AM, Rojas LJ. Can ceftazidime-avibactam and aztreonam overcome ß-lactam resistance conferred by metallo-ßlactamases in enterobacteriaceae? Antimicrob Agents Chemother 2017;61(4): e02243. DOI: 10.1128/AAC.02243-16.

18. Karaiskos I, Galani I, Souli M. Novel ß-lactam-ß-lactamase inhibitor combinations: expectations for the treatment of carbapenemresistant gram-negative pathogens. Expert Opin Drug Metab Toxicol 2019;15(2):133-149. DOI: 10.1080/17425255.2019.1563071.

19. Tumbarello M, Trecarichi EM, Corona A, De Rosa FG, Bassetti M, Mussini C, et al. Efficacy of ceftazidime-avibactam salvage therapy in patients with infections caused by Klebsiella pneumoniae carbapenemase-producing K. pneumoniae. Clin Infect Dis 2019;68(3):355-364. DOI: 10.1093/cid/ciy492.

20. Rathish B, Wilson A, Warrier A, Prakash S, Babu R, Joy S. Clinical outcomes in carbapenem-resistant Enterobacteriaceae infections treated with ceftazidime-avibactam: a single-center observational study. Cureus 2021;13(2):e13081. DOI: 10.7759/cureus.13081.

21. Nagvekar V, Shah A, Unadkat VP, Chavan A, Kohli R, Hodgar S, et al. Clinical Outcome of Patients on Ceftazidime-avibactam and Combination Therapy in Carbapenem-resistant Enterobacteriaceae. Indian J Crit Care Med 2021;25(7):780-784. 